\title{
Innovative method to measure the extent of the stable phase-space region of proton synchrotrons
}

\author{
E. H. Maclean, ${ }^{1,2}$ M. Giovannozzi, ${ }^{2, *}$ and R. B. Appleby ${ }^{3}$ \\ ${ }^{1}$ University of Malta, Msida MSD 2080, Malta \\ ${ }^{2}$ Beams Department, CERN, 1211 Geneva 23, Switzerland \\ ${ }^{3}$ The University of Manchester and Cockcroft Institute, Manchester M13 9PL, United Kingdom
}

(Received 10 January 2019; published 12 March 2019)

\begin{abstract}
The advent of circular accelerators based on superconducting magnets has revolutionized the field of beam dynamics, with particle motion turning from linear to nonlinear due to unavoidable high-order field errors generated by the ring magnets. Nonlinear dynamics was already well mastered, e.g., in the close field of celestial mechanics as similar problems had been considered and successfully tackled. Hence, several results were available to aid comprehension of the behavior of charged particle beams under the influence of nonlinear forces. Here, we discuss how concepts derived from the theory of dynamical systems, linked with the fundamental Kolmogorov-Arnold-Moser theory and Nekhoroshev theorem, can be successfully applied to the analysis of nonlinear motion of charged particles in a circular accelerator. Based on these ideas, an innovative method to measure the extent of the phase-space region within which bounded motion occurs is presented, which has been successfully tested for the first time at the CERN LHC.
\end{abstract}

DOI: 10.1103/PhysRevAccelBeams.22.034002

\section{INTRODUCTION}

Dynamic aperture (DA) is the amplitude of the phase space region where stable motion occurs. It is one of the key quantities for the design of modern colliders based on superconducting magnets, such as Tevatron [1-3], HERA [4-7], RHIC [8], the Superconducting Super Collider (SSC) $[9,10]$, and the CERN Large Hadron Collider (LHC) (see e.g., Ref. [11] for a detailed overview).

In a mathematical sense, stable motion implies bounded motion for arbitrary time. In a physical context, particle stability can be linked to a maximum number of turns $N_{\max }$ for which bounded motion occurs, where $N_{\max }$ is set on the basis of the specific application under consideration. If an ensemble of initial conditions defined on a polar grid $(x=r \cos \theta, y=r \sin \theta 0 \leq \theta \leq \pi / 2$, where $x, y$ are expressed in units of $\sigma_{x}, \sigma_{y}$ of the beam dimension) is tracked for up to $N_{\max }$ turns to assess their stability, then the DA can be defined as [12]:

$$
D(N)=\frac{2}{\pi} \int_{0}^{\pi / 2} r_{\mathrm{s}}(\theta ; N) d \theta \equiv\left\langle r_{\mathrm{s}}(\theta ; N)\right\rangle_{\theta}
$$

\footnotetext{
Corresponding author. massimo.giovannozzi@cern.ch

Published by the American Physical Society under the terms of the Creative Commons Attribution 4.0 International license. Further distribution of this work must maintain attribution to the author(s) and the published article's title, journal citation, and DOI.
}

where $r_{\mathrm{s}}(\theta ; N)$ stands for the last stable amplitude (disregarding any stable domain disconnected from the origin) for up to $N$ turns in the direction $\theta$, for $N<N_{\max }$. Given the choice of the coordinates, $D(N)$ is expressed in units of beam sigma. In this way, dynamic aperture can be considered a function of time, with an asymptotic value representing the region of stability for arbitrary time. Whenever the border of the DA is inside the phase-space region occupied by the beam, particles will be pushed toward high amplitudes and lost. It is worth recalling that more refined algorithms can be devised, where different directions in phase space have different weights [12]. Moreover, it should be mentioned that the initial values of the angular variables in each of the transverse directions are set to zero, and no scan is performed over the angles. Indeed, as shown in Ref. [12], the dynamics allows all values of the phases, as well as negative values of $x$ and $y$, to be sampled, which explains why the polar grid is limited to the first quadrant of the $x-y$ space. In this respect, the $x-y$ space is to be considered like the space of linear invariants. It is also important to stress that while Eq. (1) should be extended to $6 \mathrm{D}$ by including the longitudinal phase space to the scan procedure, a single value of the initial momentum offset is used, corresponding to the $3 \sigma$ value of the momentum distribution.

The problem of determining the DA, either analytically or numerically, has fostered knowledge transfer from other scientific fields, in particular from the domain of nonlinear dynamical systems, such as the successful introduction and application of normal forms [13-18] in the 1980s. 

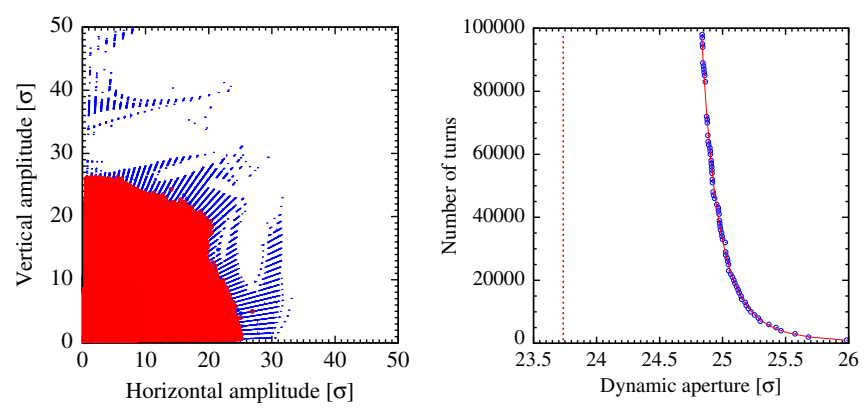

FIG. 1. Left: Dynamic aperture of a model of the LHC ring in normalized physical space. The red points represent initial conditions stable up to the maximum number of turns $\left(10^{5}\right)$. The blue points represent unstable conditions and the marker's size is proportional to the number of turns for which the motion is still bounded. Right: Time evolution of DA. The markers represent the numerical results, while the continuous line shows the fitted inverse logarithmic law, and the vertical dashed line represents $D_{\infty}$ (the fit parameters are $D_{\infty}=23.74 \pm 0.01, b=$ $0.439 \pm 0.005, \kappa=1.40 \pm 0.05$ [19]).

An accurate numerical computation of DA, as well as a good estimate of the error associated with the protocol used in the numerical simulations, is of paramount importance to ensure the reliability of DA as a figure-of-merit for assessing synchrotron performance. A general discussion of the DA definition, its computation, and accuracy can be found in Ref. [12]. Computation consists of simulating the evolution of a large number of initial conditions, distributed to provide good coverage of the phase space under study, probing whether motion remains bounded over the time interval selected for the simulations. An example of results from such a simulation is shown in Fig. 1 (left), which represents a set of initial conditions in the polar grid of normalized physical space, for one configuration of the LHC machine at top-energy for the clockwise beam (the socalled Beam 1). Red markers identify initial conditions whose motion is bounded up to $10^{5}$ turns, while blue markers identify initial conditions giving rise to unbounded motion, which eventually leads to a loss onto the mechanical aperture of the LHC. The red region provides a direct indication of the size of the DA.

Given the CPU-intense character of these simulations, studies have explored techniques for finding easy-tocompute dynamical quantities, such as Lyapunov exponents [20-24] or so-called early indicators [24], that are well correlated with the regular and bounded character of the beam dynamics. In parallel, models to fit, and eventually extrapolate, the dependence of the DA on the number of turns $[25,26]$ have been looked for. The rationale is that long-term behavior of the DA, a computationally heavy task, can be extrapolated using knowledge from numerical simulations performed over a smaller number of turns. Potentially, a large number of initial conditions can be used to improve the accuracy of numerical simulations.
Increasing the number of initial conditions has no drawbacks in terms of CPU-time needed, as parallelization over the initial conditions can be easily performed [27]. Additionally, a more efficient estimate of the long-term behavior of the DA would make easier to analyze several configurations of the circular accelerator, which is sometimes a must to gain insight in the deeper nature of the beam dynamics.

The plan of the paper is the following: in Sec. II the link between DA and losses in circular accelerators is reviewed, while in Sec. III the approaches used to measure DA are presented and discussed. In Sec. IV the new approach is presented and discussed in detail, including the results of the experimental campaign and the comparison with numerical simulations. Finally, conclusions are drawn in Sec. V.

\section{DYNAMIC APERTURE AND BEAM LOSSES}

The capability of computing the DA and its boundary is essential for modern accelerator physics. More than that, a deeper understanding of the phenomena generating the boundary between stable and unstable motion potentially opens the route to controlling DA (see, e.g., Refs. [28,29]), which may in turn provide the means to improve synchrotron performance, and well-defined criteria to specify quantitative bounds to the magnets' field quality. Such understanding and control of DA remains elusive, and is an active area of research in the domain of single-particle beam dynamics.

The answer to the quest for a model for the time-evolution of DA was provided by two fundamental results of the theory of dynamical systems, namely the Kolmogorov-ArnoldMoser (KAM) [30] and the Nekhoroshev [31] theorems. According to the results of Refs. [25,26], the following scaling law holds

$$
D(N)=D_{\infty}+\frac{b}{(\log N)^{\kappa}}
$$

where $D_{\infty}$ represents the asymptotic value of the amplitude of the stability domain. $b$ and $\kappa$ are additional parameters. In Fig. 1 (right) the behavior of $D(N)$ is shown. Dots represent the numerically-computed values of $D(N)$ according to Eq. (1), while the continuous line represents the fitted function based on Eq. (2), showing an excellent agreement with the numerical data. The dotted line represents $D_{\infty}$.

The model (2) is compatible with the hypothesis that the phase space is partitioned into two regions: a central core, with $r<D_{\infty}$, where KAM [30] surfaces bound the motion, thus producing a stable behavior apart from a set of small measure where Arnold diffusion can take place; an outer part, with $r>D_{\infty}$, where the escape rate to infinity is given by a Nekhoroshev-like estimate [31-33] 


$$
N(r)=N_{0} \exp \left[\left(\frac{r_{*}}{r}\right)^{1 / \kappa}\right]
$$

where $N(r)$ is the number of turns that are estimated to be stable for particles with initial amplitude smaller than $r$. Experience with the analysis of data from numerical simulations of various configurations of the LHC [26] and from experimental data from the Tevatron [19] showed that the fit parameters $b, \kappa, D_{\infty}$ can assume signs that go beyond what is predicted by strictly applying the model based on Nekhoroshev theorem. At this point, the scaling law for DA has been used to propose a model for the evolution of beam intensity in a hadron synchrotron [19], which is the basis of the new experimental method proposed in this article to measure DA. If the beam distribution is Gaussian in $x$ and $y$

$$
\rho_{G}(x, y)=\frac{1}{2 \pi \sigma_{x} \sigma_{y}} e^{-\left(\frac{x^{2}}{2 \sigma_{x}^{2}}+\frac{y^{2}}{2 \sigma_{y}^{2}}\right)}
$$

then after transforming to polar coordinates and applying (1), i.e., assuming that particles with amplitude beyond $D(N)$ at turn $N$ are lost, then evolution of beam intensity $I(N)$ can be found as [19]

$$
\frac{I(N)}{I(1)}=1-\int_{D(N)}^{+\infty} e^{-\frac{r^{2}}{2}} r d r=1-e^{-\frac{D^{2}(N)}{2}},
$$

where $D(N) \underset{N \rightarrow 1}{\longrightarrow}+\infty$ (as $N=1$ is assumed to be the initial turn number), and $D(N)$ is assumed to be expressed in units of sigma. Equation (5) establishes a direct link between DA and beam lifetime in a hadron synchrotron. Although Eqs. (1) and (5) have been derived for the case of equal emittances, which is the most common case for hadron rings and represent the experimental conditions of the measurements reported here, they can be generalized to the case of unequal emittances.

\section{OVERVIEW OF DA MEASUREMENT TECHNIQUES}

Measuring DA is yet another challenge. It is an important goal in itself since it a allows examination of nonlinear single-particle motion. Further, in modern synchrotrons DA is an essential parameter in the design stage. Measuring DA provides a means to confirm the design choices, and allows benchmarking of the analysis and simulations tools used. This is essential in view of the next generation of circular machines. The main results achieved in the past are reported in Refs. [7,34-36], for HERA, Tevatron, and SPS. The comparison between measurements and simulations is reviewed in Ref. [37].

More recently, with the LHC becoming operational, DA measurement has regained much attention. The first result is reported in Ref. [38], where the DA for the
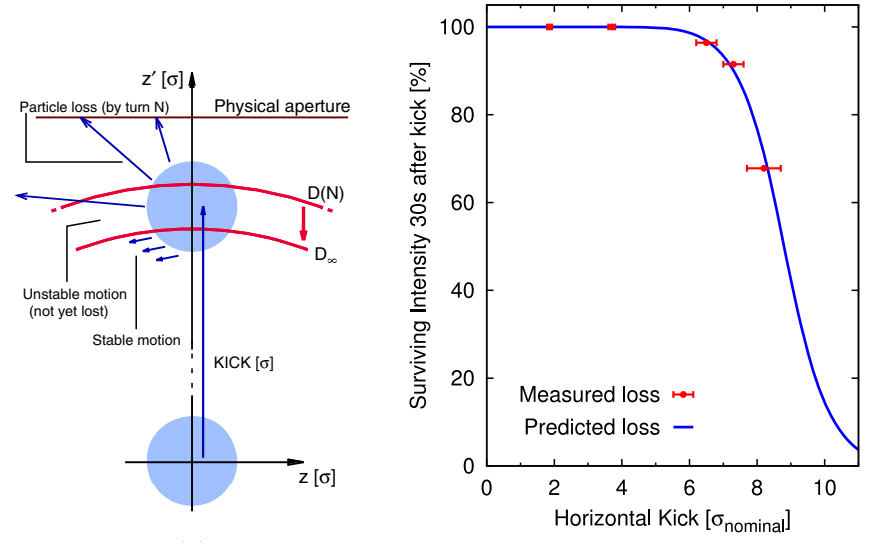

FIG. 2. Left: Sketch of the principle of the standard method to measure DA. Right: Measured and simulated losses (from Ref. [38]).

counterclockwise beam (the so-called Beam 2) has been measured using the standard method, i.e., by deflecting the beam and observing beam losses as a function of the displacement (see Fig. 2, where the good agreement between measurements and simulations is visible).

However, the standard method has some drawbacks. First, it requires deflecting the beam by an amount that is a sizeable fraction of the DA: this prevents its application at top energy in the LHC due to the strength required. Second, non-negligible, fast beam losses are intrinsic to this method: the risk of beam-induced quenches makes this feature not particularly suitable for a superconducting machine.

In the DA measurement method derived from Eq. (5) the beam is gradually and slowly blown-up simultaneously in both transverse directions by means of an appropriate excitation, typically a dipolar excitation, until some beam losses appear. Measurement of intensity decay with time then provides the needed information on DA, which is obtained by performing a fit to beam losses as a function of time. The gentle excitation does not generate large or fast losses, and no beam displacement is required as the measurement relies only on the growth of transverse beam size, avoiding the drawbacks of the standard method.

Equation (5) assumes the beam has a Gaussian distribution in both transverse directions. It is clear that the functional form of the relationship between losses and DA as expressed in Eq. (5) depends critically on the actual transverse beam distribution. Alternative expressions can be derived assuming different beam distributions, such as done in [19], where the case of quasiparabolic or LévyStudent distributions have been studied to consider the case of beam without tails or with heavy tails, respectively. Furthermore, a superposition of two or more Gaussians is often a suitable model and Eq. (5) provides a closed-form expression for the losses as a function of DA. Therefore, in a large variety of practical situations, our approach can provide a description of the measured beam losses. 


\section{THE INNOVATIVE MEASUREMENT TECHNIQUE}

\section{A. Experimental results}

The proposed technique has been used in two experimental sessions at the LHC to probe the DA of Beam 1 at injection energy $[39,40]$. The best estimate of the DA for the as-built LHC ring is known to be of the order of $11 \sigma$ ( $\sigma$ is given for the nominal LHC beam emittance, i.e., $\epsilon_{\mathrm{n}}=\beta \gamma \epsilon=3.75 \mu \mathrm{m}$, where $\beta, \gamma$ are the relativistic quantities) [41], hence, special circuits powering octupolar magnets (in total eight per ring) intended to compensate for the corresponding field error of the LHC main dipoles [11] (see also Fig. 3), have been used to shrink the DA. Clearly, this has no impact on the validity of the
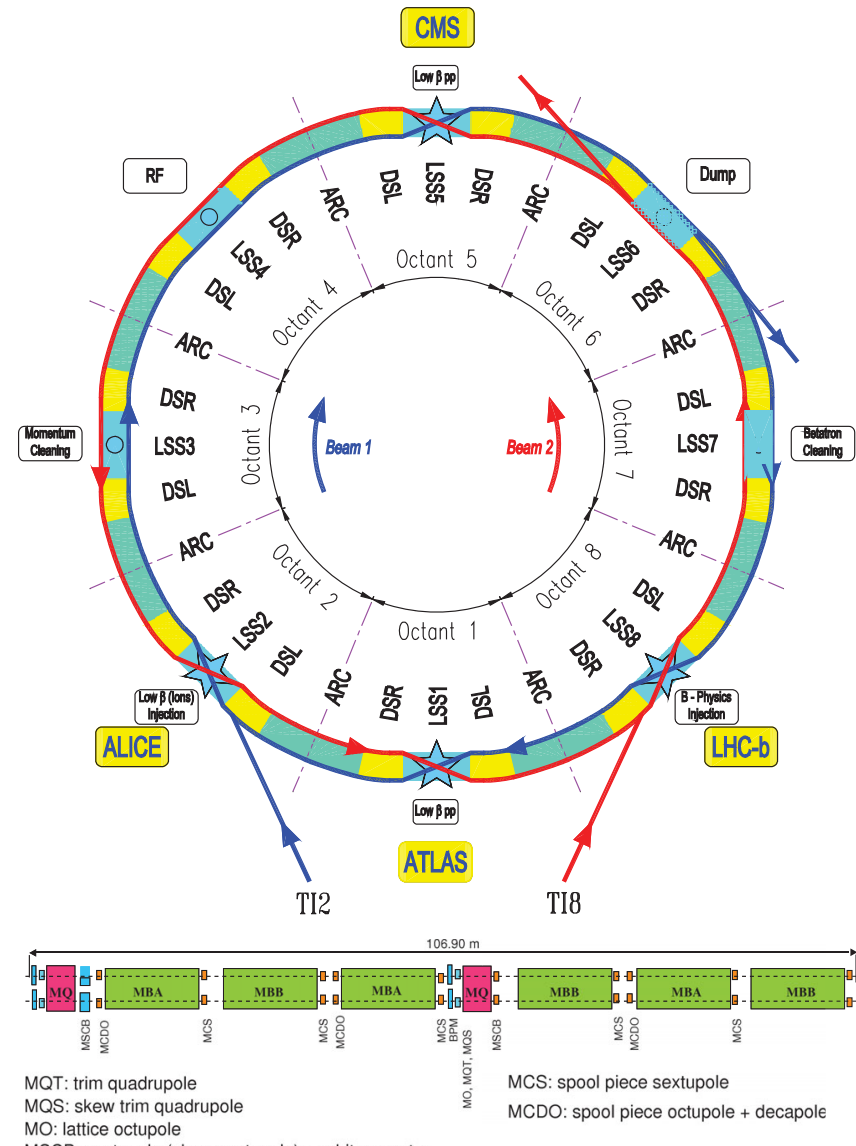

MQS: skew trim quadrup

MSCB: sextupole (skew sextupole) + orbit corrector

FIG. 3. Upper: Layout of the LHC (from Ref. [11]). The ring eight-fold symmetry is visible, together with the arcs and the long straight sections. Bottom: Layout of the LHC regular cell (from Ref. [11]). Six dipoles and two quadrupoles with the dipole, quadrupole, sextupole, and octupole magnets (for closed orbit, tune, chromaticity correction and beam stabilization, respectively) are shown. The spool pieces used to compensate the systematic $b_{3}$ component (MCS), $b_{4}$ and $b_{5}$ components (MCDO nested magnets) are also shown. The field imperfections of LHC magnets are given as $B_{y}+i B_{x}=B_{\text {ref }} \sum_{n=1}^{M}\left(b_{n}+i a_{n}\right)\left(\frac{x+i y}{R_{\mathrm{r}}}\right)^{n-1}$ where $R_{\mathrm{r}}=17 \mathrm{~mm}$. experimental technique and has several advantages: first, it provides the opportunity to probe several ring configurations, corresponding to different powering level of the octupole circuits; second, the strongly powered octupole circuits dominate the beam dynamics, generating a configuration in which a few strong and well-controlled nonlinearities are at work, rather than many weak, uncontrolled sources. This simplifies the task of benchmarking the experimental results against numerical simulations.

Two types of configurations have been considered: one with the same sign for the eight octupole circuits in Ring 1, in which the common absolute value has been varied; and one with alternating signs strength $(++--++--$ starting from the arc between ATLAS and ALICE experiments). Note that the circuit in the first arc could not be used and had to be set to zero, thus breaking the symmetry of the original scheme.

During the measurements, the collimation system is retracted to avoid any interference, and a single bunch of low intensity, typically $\sim 10^{10}$ protons, is injected, gently blown up transversely, and the intensity decay recorded. The bunch intensity is a factor of ten lower than the nominal value, which allows neglecting all intensitydependent effects, thus ensuring that the dynamics is essentially that of a single particle. Furthermore, under these well-controlled conditions the transverse distributions are to a very high degree of accuracy represented by a Gaussian, which fulfils the assumption at the heart of the new method. Moreover, the lifetime due to beam interaction with the residual gas is about $100 \mathrm{~h}$, much longer than the measurement time of the intensity decay, which is of the order of minutes. Furthermore, while the beam-gas interaction generates a slow and amplitude-independent transport of charged particles towards higher amplitudes, the nonlinear effects become stronger as particle's amplitude grows until they become the dominant source of amplitude growth. This approach is repeated for different values of the strength of the octupole circuits, which are called MCO (for magnet corrector octupole) according to the official LHC nomenclature. Each configuration probed during the experiment is labeled with $\sum K_{\mathrm{MCO}}=$ $N_{\mathrm{MCO}} e / p\left(\partial^{3} B_{y} / \partial x^{3}\right), \quad p / e$ being the beam magnetic rigidity, $B_{y}$ the vertical magnetic field component, and $N_{\text {MCO }}$ the total number of MCO magnets in Ring 1.

The whole sequence of measurements is shown in Fig. 4, where the evolution of beam intensity and MCO magnetic strength is plotted against time. Once a sizeable intensity change is obtained the octupoles' strength is varied and this is repeated for several magnetic configurations of the LHC lattice. It is worth noting that occasionally a new bunch had to be injected, which required new excitation to increase the transverse beam size. Transverse distributions of the bunch were recorded to provide information about the beam size, needed to normalize the beam losses. The configurations considered in the analysis presented in this paper are those 


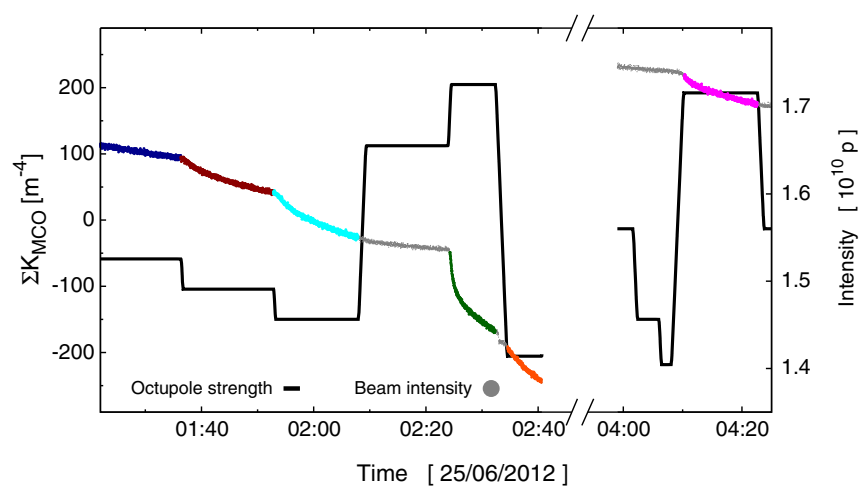

FIG. 4. Sequence of measurements performed during the experimental session. The evolution of the beam intensity and of the MCO magnets' strength is shown. The color is used to indicate the data selected for the analysis presented in the rest of the paper.

for which measurable beam losses have been recorded, and the intensity data have been considered only for the time intervals corresponding to constant strength of the $\mathrm{MCO}$ magnets.

A summary of the measurements of the intensity decay over time is shown in Fig. 5, where the evolution of the relative beam intensity is plotted for the different configurations of MCOs strength. The value of $D(N)$ is estimated by applying Eq. (5) to the experimental data.

\section{B. Comparison with numerical simulations}

Numerical simulations are performed for the configuration probed during the experiment using the SixTrack code [42], which implements a second-order symplectic integration of the equations of motion in the $6 \mathrm{D}$ phase space. The LHC model is based on the linear ring lattice to which the measured field quality of the various classes of magnets are added as thin-lens multipoles, from order sextupole to twenty-two-pole [41]. The uncertainties affecting the field quality measurements are taken into account by generating sixty realizations of the complete set of magnetic field

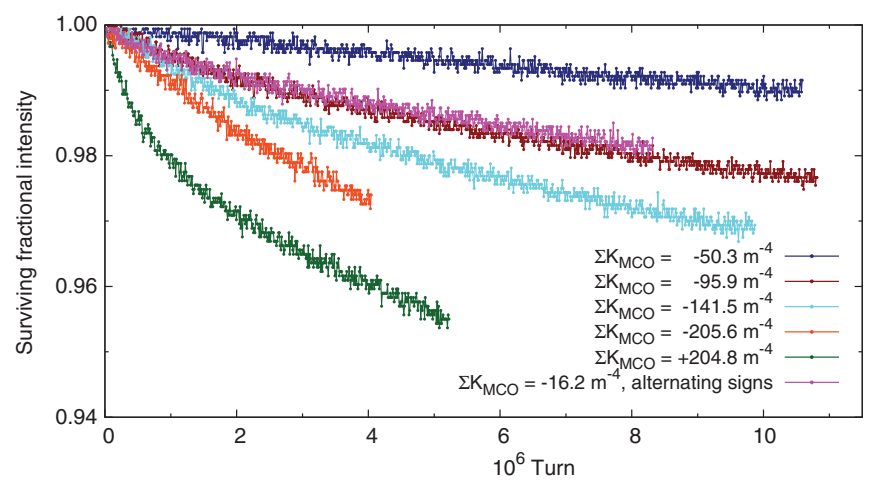

FIG. 5. Relative beam intensity vs turns plotted for all octupolar configurations probed in the LHC DA experiment with Beam 1. The different intensity decay rate is visible. errors for the LHC, such that a single DA simulation consists, in fact, of sixty cases corresponding to the different realizations. The model is further improved by including ad hoc errors computed to reproduce the measured closed orbit (dipole errors), the linear coupling (skew quadrupole errors), and the variation of frequency in phase space with amplitude (octupole errors). A detailed analysis showed that the DA is almost independent on the presence of realistic closed-orbit effects, while it is much more sensitive to linear coupling [43].

Initial conditions for $x$ and $y$ are distributed in phase space on a polar grid made of 59 angles, and a uniform radial distribution with 30 pairs of initial conditions every $2 \sigma$ in amplitude, while the angular variables are set to zero [12]. The initial momentum offset is set to $3 \times 10^{-4}$, equal to $3 \sigma$ of the momentum distribution.

A massive campaign of numerical simulations aimed at studying all experimental machine configurations has been carried out using the volunteer-based computing platform LHC@home (see Ref. [44] and references therein). As a result, $D(N)$ is shown in Fig. 6 comparing experimental and numerical results for three configurations of $\mathrm{MCO}$ strength. The curves correspond to the numerical results for the sixty realizations, which generate an uncertainly on the DA value of about $\pm 0.5 \sigma$. This is larger than the absolute accuracy due to the grid of initial conditions that is on the order of $0.1-0.2 \sigma$. It is worth stressing that $D(N)$ is always expressed in terms of nominal $\sigma$, which is the value obtained by using the nominal value of the normalized beam emittance, i.e., $3.75 \mu \mathrm{m}$, and this value is different with respect to that measured during the experimental session.

The agreement between numerical and experimental results is apparent, the experimental data always being within the range of DA values provided by the sixty

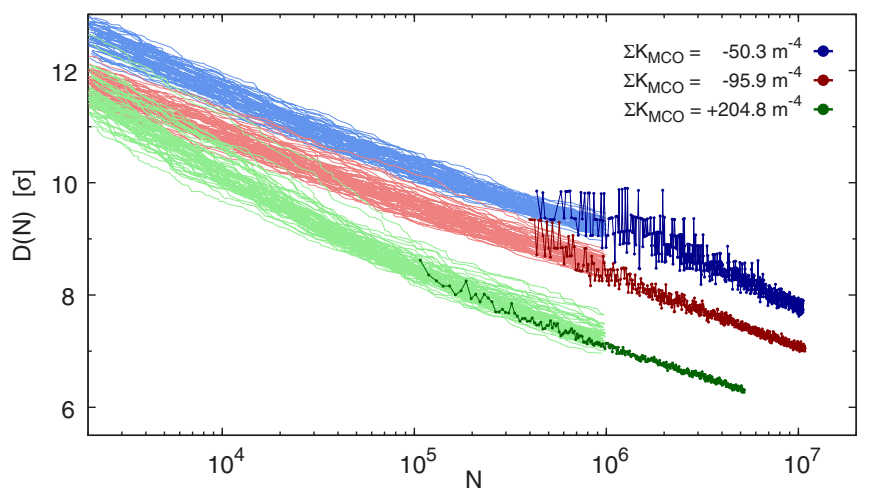

FIG. 6. DA estimates from simulations (lines) and measurements (lines with full markers) for three selected cases. The excellent agreement is visible, even if some difference in slope between measured and simulation results is visible for the case $\Sigma K_{\mathrm{MCO}}=-50.3 \mathrm{~m}^{-4}$. The DA is not given in units of beam $\sigma$, but rather in units of the nominal $\sigma$ obtained with the nominal beam emittance of $\epsilon_{\mathrm{n}}=3.75 \mu \mathrm{m}$. 

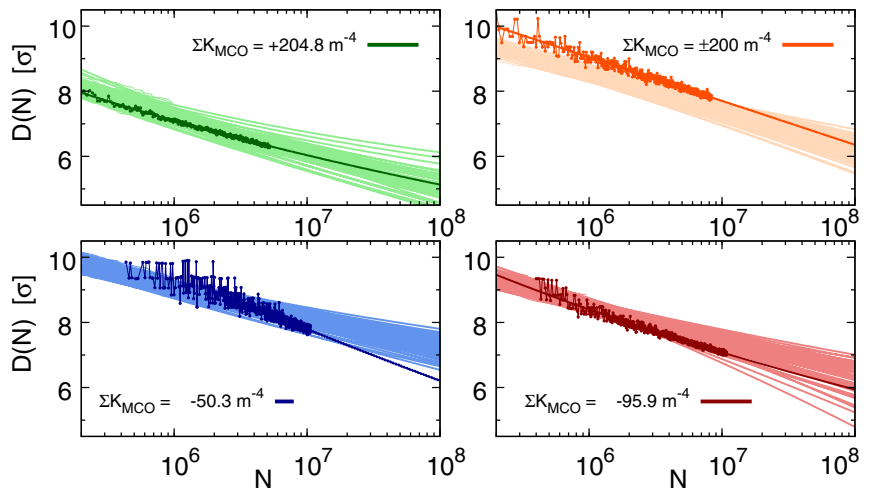

FIG. 7. Comparison between measured DA (lines with full markers) and extrapolation (lines) of the numerical estimate based on the scaling law (2) for four selected cases. The very good agreement is clearly visible. The upper right plot refers to the case with alternating signs for the strength of the MCOs. The DA is not given in units of beam $\sigma$, but rather in units of the nominal $\sigma$ obtained with the nominal beam emittance of $\epsilon_{\mathrm{n}}=3.75 \mu \mathrm{m}$.

realizations. Some difference in the slope of $D(N)$ is visible only for the case with $\Sigma K_{\mathrm{MCO}}=-50.3 \mathrm{~m}^{-4}$. Figure 7 shows the comparison between the measured DA and the extrapolation of the results of numerical simulations based on the scaling law (2). The results of numerical simulations have been fitted using the scaling law (1) using the data for $N \leq 10^{5}$ and the fitted functions for the sixty realizations have been used to extrapolate the DA up to $N=10^{8}$. In addition to the three configurations shown in Fig. 6, a fourth one with alternating sign strengths of MCOs is plotted: a very good agreement between measurements and extrapolations is visible. The standard protocol for the computation of the DA for LHC is based on the evaluation of the boundedness of the particle's motion over $10^{5}$ or $10^{6}$ turns, the latter value being used in the presence of beambeam interactions [11]. It is worth stressing that the scaling law (2) allows useful extrapolation of the DA over a time interval that is one or two orders of magnitude longer than that used to perform the numerical computations.

A summary plot comparing all beam measurements and the results of numerical simulations is shown in Fig. 8, where the DA values correspond to $D\left(1 \times 10^{6}\right)$ turns.

The error bars associated with the results of numerical simulations are obtained from the DA distribution of the sixty realizations, while those for the experimental results are given by the spread of the measured $D(N)$ close to $N=10^{6}$ (see Fig. 6). Globally a very good agreement is found, with most of the configurations featuring measurements and numerical simulations compatible within the estimated error bars. The configuration corresponding to the smallest MCO strength shows the largest disagreement between measurement and simulations. However, it should be noted that the difference is at most at the level of $20 \%$. All these results indicate a very good agreement between

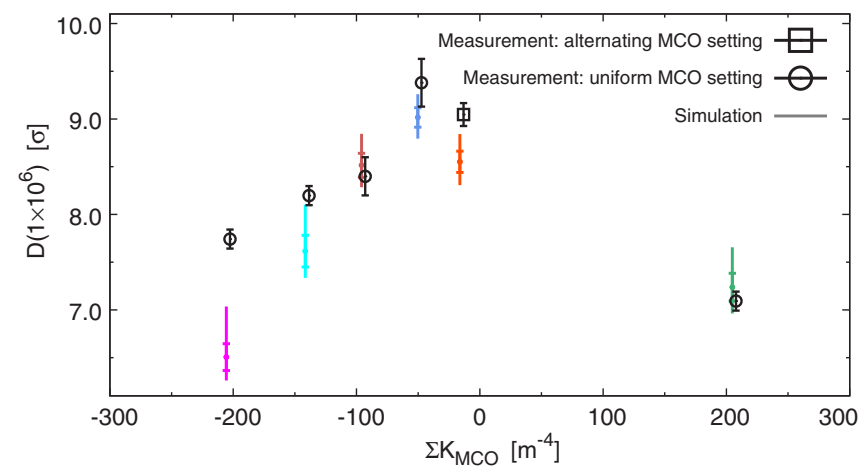

FIG. 8. Summary of the measured and simulated DA values corresponding to $D\left(1 \times 10^{6}\right)$, plotted for the various configurations of the MCO strength. The error bars of measurement results are obtained by the spread of DA values for $10^{6}$ turns (see the experimental curves in Fig. 6). The error bars of simulation results are obtained from the DA distribution over the sixty realizations of the LHC lattice (both $\mathrm{rms}$ and $\mathrm{min} / \mathrm{max}$ error bars are shown). The DA is not given in units of beam $\sigma$, but rather in units of the nominal $\sigma$ obtained with the nominal beam emittance of $\epsilon_{\mathrm{n}}=3.75 \mu \mathrm{m}$.

the measurements, based on the new method, and the numerical simulations, thus suggesting to move forward and probe new configurations.

The LHC at top energy is an additional test case of particular interest, not only for the sake of improving our general understanding of nonlinear beam dynamics, but also for a direct measurement of the impact on DA of the highorder, nonlinear correctors installed in the triplet quadrupoles of the LHC experimental insertions. These special magnets have the function to mitigate the harmful effects of nonlinear field errors stemming from the strong quadrupoles, located close to the interaction point. In the LHC, the combined effect of magnets' misalignments and measured triplets' field quality is such that it is not possible to provide reliable information to compute the correctors' optimal strength. Beam-based measurements are therefore mandatory, yet extremely difficult and time-consuming to perform [45]. Moreover, the physical observables probed in these experiments are only indirectly linked with DA, the ultimate figure-of-merit. The demonstrated method provides a straightforward means to assess the size of DA, impossible using conventional techniques, including its dependence on the nonlinear corrector magnets. Computing the optimal strength of these high-order, nonlinear correctors is anticipated to be vital for the planned high-luminosity upgrade of the LHC [46], for which the performance goal means accurate control of nonlinear effects is even more essential than for the LHC. This method has the potential to provide the needed performance.

\section{CONCLUSIONS}

In this paper, ideas from the theory of dynamical systems have been used to analyze the problem of nonlinear motion 
of protons in circular accelerators such as the LHC. The understanding of such motion is crucial to the design and performance optimization of current and future accelerators, and accurate computation of dynamics aperture is of central importance for ensuring synchrotron performance.

A solid framework based on fundamental theorems has been built in recent years and the experimental observations presented in this paper represent a serious test of such a framework. Several configurations of the LHC ring have been probed in a dedicated experimental session and their detailed analysis, together with a massive campaign of numerical simulations, allows the conclusion that the proposed framework works very well in reproducing the behaviour of measured beam losses. Furthermore, the good agreement between measurements and simulations means the proposed method to measure the DA is indeed feasible and very valuable. Note that the new method overcomes a number of serious limitations of the standard one, which are affecting particularly high-energy, superconducting rings.

It is worth stressing that the good agreement between measurements and simulation results reported in this paper is an essential outcome our studies, as it provides a serious benchmark of concepts and tools. Indeed, it is useful to recall that for past superconducting rings, the agreement between measurements and simulations was at the level of a factor of two, whereas we show that such a discrepancy can be reduced to about $20 \%$. The relevance of benchmarking tools and concepts should not be underestimated, as this is an essential point when undertaking studies for future machine, where only numerical simulations can be used to optimize their performance.

As a next step, it is planned to pursue the experimental efforts in the direction of exploring more LHC ring configurations, such as top energy with squeezed optics, as well as performing loss measurements over longer timescales. The first point allows probing more configurations, thus providing even more confidence in the proposed approach. Assessing the beam behavior over longer timescales allows a detailed study of the predictiveness of our approach, which is ultimately the key aspect of our proposal. In fact, the essence of our method relies on the evaluation of the scaling law of the DA, rather than considering a single value corresponding to a fixed and given number of turns.

The predictiveness analysis might lead to a revision of the maximum number of turns, which are currently assumed as $10^{5}$ (without beam-beam effects) or $10^{6}$ (with beam-beam effects), used in the DA tracking studies. Eventually, optimization of performance of future colliders should be based on the analysis of expected beam losses over time rather than on plain DA computed for a given and fixed number of turns.

\section{ACKNOWLEDGMENTS}

One of the authors (M.G.) would like to thank A. Bazzani for several fruitful discussions. The
LHC@home volunteers are warmly thanked as their CPU-time contribution was of invaluable help for the massive tracking campaigns.

[1] N. M. Gelfand, in Proceedings of SSC Workshop on Accelerator Physics Issues for a Superconducting Super Collider, Ann Arbor, MI, USA, 1983, edited by M. Tigner (Michigan Trout Unlimited, Ann Arbor, MI, 1984), p. 190.

[2] V. Visnjic, in Proceedings of 1991 Particle Accelerator Conference, edited by J. Chew and L. Lizama (IEEE, Piscataway, NJ, 1991), p. 1701.

[3] V. Visnjic, in Proceedings of Workshop On Nonlinear Problems In Future Particle Accelerators, edited by W. Scandale and G. Turchetti (World Scientific, Singapore, 1991).

[4] R. Brinkmann and F. Willeke, DESY Report No. DESYHERA-88-08, 1988.

[5] F. Zimmermann and F. Willeke, DESY Report No. DESYHERA-91-08, 1991.

[6] F. Zimmermann, SLAC Report No. SLAC-PUB-6458, 1994.

[7] O.S. Brüning et al., Comparison of measured and computed dynamic aperture for the SPS and the HERA proton ring, Part. Accel. 54, 223 (1996).

[8] Y. Luo et al., in Proceedings of 2007 Particle Accelerator Conference, edited by C. Petit-Jean-Genaz (IEEE, Piscataway, NJ, 2007), p. 4363.

[9] T. Sen, A. Chao, and Y. Yan, in Proceedings of 1991 Particle Accelerator Conference, edited by L. Lizama and J. Chew (IEEE, Piscataway, NJ, 1991), p. 345.

[10] L. Schachinger and Y. Yan, SSC Laboratory Report No. SSC-N-664, 1989.

[11] O. Brüning et al., CERN Report No. CERN-2004-003-V-1, 2004.

[12] E. Todesco and M. Giovannozzi, Dynamic aperture estimates and phase-space distortions in nonlinear betatron motion, Phys. Rev. E 53, 4067 (1996).

[13] D. R. Douglas and A. J. Dragt, MARYLIE: The maryland lie algebraic transport and tracking code, IEEE Trans. Nucl. Sci. 30, 2442 (1983).

[14] A. J. Dragt, Elementary and advanced Lie algebraic methods with applications to accelerator design, electron microscopes, and light optics, Nucl. Instrum. Methods Phys. Res., Sect. A 258, 339 (1987).

[15] A. J. Draft, F. Neri, G. Rangarajan, D. R. Douglas, L. M. Healy, and R. D. Ryne, Lie algebraic treatment of linear and nonlinear beam dynamics, Annu. Rev. Nucl. Part. Sci. 38, 455 (1988).

[16] A. Bazzani, P. Mazzanti, G. Servizi, and G. Turchetti, Normal forms for Hamiltonian maps and nonlinear effects in a particle accelerator, Nuovo Cimento Soc. Ital. Fis. 102B, 51 (1988).

[17] E. Forest, M. Berz, and J. Irwin, Normal form methods for complicated periodic systems, Part. Accel. 24, 91 (1989).

[18] A. Bazzani, G. Servizi, E. Todesco, and G. Turchetti, Normal forms and invariants in the description of a 
magnetic lattice, Nucl. Instrum. Methods Phys. Res., Sect. A 298, 102 (1990).

[19] M. Giovannozzi, Proposed scaling law for intensity evolution in hadron storage rings based on dynamic aperture variation with time, Phys. Rev. ST Accel. Beams 15, 024001 (2012).

[20] M. Hénon and C. Heiles, The applicability of the third integral of motion: Some numerical experiments, Astron. J. 69, 73 (1964).

[21] C. Froeschlé, Numerical study of a four-dimensional mapping, Astron. Astrophys. 16, 172 (1972).

[22] G. Benettin, L. Galgani, A. Giorgilli, and J.-M. Strelcyn, Lyapunov Characteristic Exponents for smooth dynamical systems and for hamiltonian systems; A method for computing all of them. Part 2: Numerical application, Meccanica 15, 21 (1980).

[23] F. Schmidt, F. Willeke, and F. Zimmermann, Fast indicators of long-term stability, Part. Accel. 35, 249 (1991).

[24] E. Todesco, M. Giovannozzi, and W. Scandale, Prediction of long-term stability in large hadron colliders, Part. Accel. 55, 273 (1995).

[25] M. Giovannozzi, W. Scandale, and E. Todesco, Dynamic aperture extrapolation in presence of tune modulation, Part. Accel. 56, 195 (1996).

[26] M. Giovannozzi, W. Scandale, and E. Todesco, Dynamic aperture extrapolation in the presence of tune modulation, Phys. Rev. E 57, 3432 (1998).

[27] M. Giovannozzi and E. McIntosh, Development of parallel codes for the study of nonlinear beam dynamics, Int. J. Mod. Phys. C 08, 155 (1997).

[28] W. Wan and J. R. Cary, Increasing the Dynamic Aperture of Accelerator Lattices, Phys. Rev. Lett. 81, 3655 (1998).

[29] W. Wan and J. R. Cary, Method for enlarging the dynamic aperture of accelerator lattices, Phys. Rev. ST Accel. Beams 4, 084001 (2001).

[30] C. L. Siegel and J. Moser, Lectures in Celestial Mechanics (Springer Verlag, Berlin, 1971).

[31] N. Nekhoroshev, An exponential estimate of the time of stability of nearly-integrable hamiltonian systems, Russ. Math. Surv. 32, 1 (1977).

[32] A. Bazzani, S. Marmi, and G. Turchetti, Nekhoroshev stability estimates for symplectic maps and physical applications, Cel. Mech. 47, 333 (1990).

[33] G. Turchetti, in Proceedings of Number Theory and Physics, edited by J. M. Luck, P. Moussa, and M. Waldschmidt,
Springer Proceedings in Physics (Springer Verlag, Berlin, 1990), Vol. 47, p. 223.

[34] T. Collins et al., in Proceedings of the 1984 Snowmass Workshop on Design and Utilization of the Superconducting Super Collider, Snowmass, Colorado, 1984, edited by R. Donaldson and J. G. Morfin (Division of Particles and Fields of the American Physical Society, New York, 1984), p. 361 .

[35] J. M. Peterson et al., in Proceedings of the 1988 European Particle Accelerator Conference, edited by S. Tazzari (World Scientific, Singapore, 1988), p. 266.

[36] W. Fischer, M. Giovannozzi, and F. Schmidt, Dynamic aperture experiment at a synchrotron, Phys. Rev. E 55, 3507 (1997).

[37] F. Willeke, in Proceedings of 1995 Particle Accelerator Conference (IEEE Service Center, Piscataway, 1996), p. 2747.

[38] E. H. Maclean, R. Tomás, F. Schmidt, and T. H. B. Persson, Measurement of nonlinear observables in the Large Hadron Collider using kicked beams, Phys. Rev. ST Accel. Beams 17, 081002 (2014).

[39] M. Albert et al., in Proceedings of the 3rd International Particle Accelerator Conference, New Orleans, LA, 2012, edited by C. Eyberger and F. Zimmermann (IEEE, Piscataway, NJ, 2012), p. 1362.

[40] M. Giovannozzi et al., in Proceedings of the 4th International Particle Accelerator Conference, IPAC2013, Shanghai, China, 2013, edited by Z. Dai, C. Petit-Jean-Genaz, V. R. W. Schaa, and C. Zhang (JACoW, Shanghai, China, 2013), p. 2606.

[41] S. Fartoukh and M. Giovannozzi, Dynamic aperture computation for the as-built CERN Large Hadron Collider and impact of main dipoles sorting, Nucl. Instrum. Methods Phys. Res., Sect. A 671, 10 (2012).

[42] http://sixtrack.web.cern.ch/SixTrack/.

[43] E. H. Maclean et al. (to be published).

[44] J. Barranco et al., LHC@Home: a BOINC-based volunteer computing infrastructure for physics studies at CERN, Open Eng. 7, 378 (2017).

[45] E. H. Maclean, R. Tomás, M. Giovannozzi, and T. H. B. Persson, First measurement and correction of nonlinear errors in the experimental insertions of the CERN Large Hadron Collider, Phys. Rev. ST Accel. Beams 18, 121002 (2015).

[46] G. Apollinari et al., CERN Report No. CERN-2015-005. 